\title{
CEVICHE FRAMEWORK FOR DYNAMIC ADAPTATION BUSINESS PROCESS USING COMPLEX EVENT PROCESSING
}

\author{
NikaleChandrika $S^{1}$, NikamDivya $\mathrm{J}^{2}$, BhagwatPrachi $\mathrm{A}^{\mathbf{3}}$, DakaleDipali $\mathrm{S}^{\mathbf{4}}$ \\ ${ }^{1}$ Student, Computer Engineering, SND college of EngineeringYeola,SPPU, Maharashtra, India \\ nikalechandrika@rediffmail.com \\ ${ }^{2}$ Student, Computer Engineering, SND college of EngineeringYeola,SPPU, Maharashtra, India \\ divya.nikam1994@gmail.com \\ ${ }^{3}$ Student, Computer Engineering, SND college of EngineeringYeola, SPPU, Maharashtra, India \\ ,prachi.bhagwat141@gmail.com, \\ ${ }^{4}$ Student, Computer Engineering, SND college of EngineeringYeola,SPPU, Maharashtra, India \\ dakaledipali@gmail.com
}

Guided By: Prof. Kumbharde M.V., Assistant Prof. Computer Engineering, SND college of EngineeringYeola, SPPU, Maharashtra, India

\begin{abstract}
In today's Quality of Service (QoS) is main issue for the Business world,it continuously required updation for business process and various new ideas to keep a competitive high level. There is need for adaptation of business process.In this paper we propose a framework CEVICHE(Complex Event Processing for Context_adaptive processes in pervasive and Heterogeneous Environment), which provides the dynamically adaptation for improving the Quality of Service for business process adaptation.The business process generates lot of the data at the execution time and the information of the intermediate activities. The main purpose for using CEVICHE is creating context-aware business processes which are adapt dynamically in order to responding to different scenarios, and it avoids the overloading on the application. CEVICHE framework combines the the strengths of complex event processing and dynamic business process.we use complex event processing for obtaining information to improve business process and it provides the dynamic adaptation business process run time it the execution time.
\end{abstract}

Keywords:QoS , Dynamic-adaptation,Complex Event Processing,AO4BPEL,Business Process

\section{INTRODUCTION}

There is need to continuous adaptation for business process to keep higher competitive level. In this paper we creating a CEVICHE framework for dynamically adapting the business process. By combining Business Process Execution Language(BPEL) and Aspect Oriented Programming we can introducing this framework .

The Complex Event Processing(CEP) technique is use to get the information which is needed for adaptation of business process. The complex events are taking for adapting the business process dynamically and it shows the real-time relationships between different events. For monitoring theQoS ,require the alert to administrator when the running process not responding,for adapting automatic ally responsding business process We developing CEVICHE framework. It provides run-time dynamic adaptation business process during the execution time and CEVICHE relies on BPEL and it is a most comman orchestration language.In this system using the aspect oriented programming which is specially use for inheriting the result from other programming approaches, such as reflection,openimplementation ,meta-object protocols and generative programming.It is uses for improving the software system's concerns and add the crosscutting functionality without changing the logic of business process of software.AOP apply on compile time or run time to provede a flexibility

\section{RELATED WORK}

In paper [1] "Using Complex Event Processing for Dynamic Business Process Adaptation" By author Gabriel Hermosillo, Lionel Seinturier, Laurence Duchene, INRIA Lille Nord Europ-Univercity of Lille1 Laboratoire LIFL-CNRS UMR 8022 Lille,France.In this they described how the real time business process is adapt dynamically using Complex Event Processing. The On Line Car Rental System is define to show the scenario of the framework which is used in this paper.The CEVICHE framework improves the Quality of Service.

In paper [2] "A Highly Accurate Prediction Algorithm for Unknown Web Service QoS Values" By author You Ma, Shangguang Wang, , member of IEEE, Patrick C.K. Hung, Member of IEEE, Ching-Hsien Hsu, Member of IEEE, Qibo Sun and Fangchun Yang, Senior Member, IEEE, They presented in this paper the HAPA algorithm which is CFbased algorithm used for the purpose of the quality of Service. Two types of HAPA are defined this are User Based HAPA and Item Based Hapa for the prediction of QoS for the unknown web services.

In paper [3] "A Pattern-Based Architecture for Dynamically 
Adapting Business Processes" By author Mohamed LamineBerkane, Lionel Seinturier, Mahmoud Boufaida, LIRE Laboratory, sMentouri University of Constantine, Algeria.In this paper the patterns based architecture is present for designing the adaptation of business process. The patterns are used to provide feasible solution to comman system problems. In this paper the TRAP or BPEL framework are introduced for providing dynamic adaptation of business process.

In paper [4] "Event Driven Approach for Dynamic Business Process Adaptation" By author Ram Pradhan, Department of Computer Science, P.E.S. Modern College of Engineering, Pune,ISSN: 2248-9622 International Conference on Industrial Automation and Computing (ICIAC). In this paper author presents Dynamic Adaptation Framework(DAF), for combining strength of complex event and dynamic business process adaptation this DAF framework is used.

In paper [6] “ The Undoing Event Driven Adaptation of Business Processes" By author SébastienMosser, Gabriel Hermosillo, Anne-Françoise Le Meur, Lionel Seinturier Laurence DuchienINRIA Lille Nord Europe University of Lille 1 Laboratoire LIFL - CNRS UMR 8022Lille, France. It involves proper solution for adapting business process, they proposed main objective of this paper is to find effective solution for the Bpunadaptation,Adapt the process using context approach for complex event processing.

\section{EXISTING SYSTEM}

In existing system, there is traditional framework in which number of categories are there and each category having a no of events run at the background of each category. In this system there should be maximum chances to failure of site due to overloading.

\section{SURVEY EXTRACTION}

In existing system unnecessary events are generated hence this system was very complex to handle. But in this proposed system we reducing the unnecessary events , avoiding the overloading and providing the good quality of services (QoS) to the user.

\section{PROPOSED SYSTEM}

In proposed system we creating CEVICHE Framework for context aware business processing.In this system, we introducing framework which help for a fast businessprocessing. In any business process users requires highly updated in every process. This business process is reducing the users time and fast accessing and provide better QoS. CEVICHE works on BPEL, it is most common orchestration language. It focuses on flow of control and different services of business process.BPEL uses web services to communicate with different partners in business process. For showing the result of this framework we take the example of Book Purchasing System in this system the given framework is use for discarding the unnecessary events which are creating at the backend in the Book Purchasing system.by using the AOP and BPEL the
CEVICHE works.In book purchasing system the book is purchasing by selecting number of categories and each category have thousands of different events at back, The CEVICHE framework is able to automatically give priority to all events and only higher priority events are executed.for creating the automatic adaptation the CEVICHE framework is used.

\section{SYSTEM ARCHITECTURE}

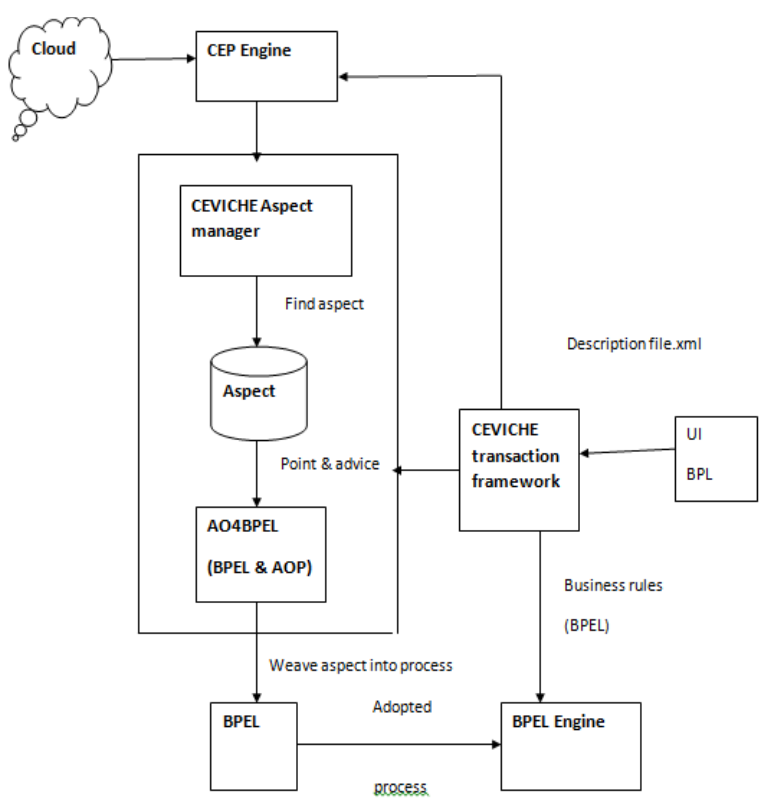

Fig -1: System Architecture

CEVICHE is a framework that facilitate integration of CEP into the business processes for dynamically adapting different circumstances. In CEVICHE Framework we define four different issues: Adaptation, Dynamicity, Integration for business process and Non-dependency for specific CEP engine.

Adaptatiion: In first issue we use the Aspect Oriented Programming (AOP) for adding and changing services frombusiness process. For this purpose AOP wants to know the part of business process which know how to perform the adaptation. In this system we use the Aspect Manager which can be use to analyzing the events with CEP and finding the alternative solution for minimizing the time and provide the better QoS which is called as point cut and advice. In this system we use wrapping for integreating the BPEL with AOP, for this purpose AO4BPEL freamwork which has ability to weave aspect at run time for the business process. CEVICHE able to work with any available CEP engine.

\section{CONCLUSION}

We have to propose the CEVICHE Frameworkfor integrating the Complex Event Processing (CEP) into the business processes for dynamically adapting different circumstances. In CEVICHE Framework we define four different issues: Adaptation, Dynamicity, Integration for business process and Non-dependency for specific CEP engine. In this CEVICHE framework propose AO4BPEL which is combination of Aspect Oriented Programming 
(AOP) and Business Process Execution Language (BPEL), that allow to user to include adaption points and condition for creating dynamically adaptable business process. CEVICHE use for monitoring the Quality of Service (QoS) of business process and adapt this process dynamically to keep competitive lavel whenever QoSdroped. For implementating this system, it is a research work in documentation phase-I and still we are working on implementation of actual system in phase-II.

\section{REFERENCES}

[1].Gabriel Hermosillo,Lionel, "Using Complex Event Processing for Dynamic Business Process Adaptation" Seinturier,LaurenceDuchien INRIA Lille Nord EuropUnivercity of Lille 1 Laboratoire LIFL-CNRS UMR 8022 Lille,France(SCC 2013)466 -473.

[2].You Ma, Shangguang Wang, A Highly Accurate Prediction Algorithm forUnknown Web Service QoS Values Member, IEEE, Patrick C.K. Hung, Member, IEEE, ChingHsien Hsu, Member, IEEE, Qibo Sun, and Fangchun Yang, Senior Member, IEEE.

[3].Mohamed Lamine Berkane1 Lionel Seinturier2 Mahmoud Boufaida1 1 LIRE Laboratory Mentouri University of Constantine, Algeria \{ml.berkane,mboufaida\}@umc.edu.dz 2LIFL-INRIA ADAM University of Lille, 59655 Villeneuve d'Ascq, France(2012).

[4]. Gabriel Hermosillo, Lionel Seinturier, Laurence Duchien" Complex Event Processing for Context-Adaptive Business Processes'’INRIA Lille - Nord Europe - University Lille 1 - Laboratoire LIFL - CNRS UMR 8022.

[5]. W. M. P. Van Der, Hofstede, A. H. M., \&Weske, M. (2003). Business Process Management : A Survey. Business, 2678(1), 1-12. doi:10.1007/3-540-44895-0_1.

[6]. SébastienMosser, Gabriel Hermosillo, "Undoing EventDriven Adaptation of Business Processes", Anne-Françoise Le Meur, Lionel

[7] J. Cardoso, A. Sheth, J. Miller, J. Arnold, and K. Kochut,"Quality of service for workflows and web service processes,"Web Semantics: Science, Services and Agents on the WorldWide Web, vol. 1, no. 3, pp. 281 - 308, 2004.

[8] M. C. Jaeger, G. Rojec-Goldmann, and G. Muhl, "Qosaggregationfor web service composition using workflow patterns,"inEDOC '04: Proceedings of the Enterprise DistributedObject Computing Conference, Eighth IEEE International.IEEE Computer Society, 2004, pp. 149-159.

[9] H.-C. Wang, C.-S.Lee, and T.-H. Ho, "Combining subjectiveand objective qos factors for personalized web service selection,"Expert Systems with Applications, vol. 32, no. 2 , pp.

$571-584,2007$.

[10] L. Zeng, B. Benatallah, A. H.H. Ngu, M. Dumas,

J. Kalagnanam, and H. Chang, "Qos-aware middleware for web services composition," IEEE Trans. Softw. Eng., vol. 30,no. 5, pp. 311-327, 2004. 